\title{
miR-26b regulates cell proliferation and apoptosis of CD117+CD44+ ovarian cancer stem cells by targeting PTEN
}

\author{
Zubiao Gao, ${ }^{1}$ Xiaofeng Ye, ${ }^{1}$ Anne Bordeaux, ${ }^{2}$ Stanka Hettich, ${ }^{3}$ Siyao Lin, ${ }^{1}$ Fang Han, ${ }^{1}$ Yan Jia ${ }^{4}$ \\ ${ }^{1}$ Department of Obstetrics and Gynecology, Foshan Chancheng Central Hospital, Foshan, Guangdong, China \\ ${ }^{2}$ Department of Pathology, Medical center, University of Freiburg, Baden-Wuerttemberg, Germany \\ ${ }^{3}$ Department of Obstetrics and Gynecology, Medical Center, University of Freiburg, Baden-Wuerttemberg, Germany \\ ${ }^{4}$ Department of Reproductive Immunology, Chengdu Xi' nan Gynecology Hospital, Chengdu, Sichuan, China
}

\begin{abstract}
Ovarian cancer (OC) is the one of the most common cancer in women globally. However, it still represents the most dangerous gynecologic malignancy even with the advances in detection and therapeutics. Thus, there is an urgent need in finding more effective therapeutic options for OC patients including cancer stem cells (CSCs). MicroRNAs (miRNAs) are small, endogenous, and non-coding RNAs that play critical roles in the progression of various types of tumor. Our aim of this study was to find the regulatory function of microRNA26 (miRNA-26b) on the cell proliferation and apoptosis of ovarian CSCs. Our studies show that miR-26b is under-regulated in human $\mathrm{CD} 117^{+} \mathrm{CD} 44^{+}$ovarian CSCs. The miR-26b overexpression inhibits the cell proliferation and promotes cell apoptosis. Moreover, phosphatase and tensin homolog (PTEN) is found to be a functional target of miR-26b. Moreover, PTEN overexpression reversed the effects of miR-26b on cell proliferation and apoptosis. PTEN overexpression remarkably accelerated cell proliferation, and inhibited cell apoptosis. These results indicate that miR-26b regulates cell proliferation and apoptosis of $\mathrm{CD} 117^{+} \mathrm{CD} 44^{+}$ovarian CSCs by targeting PTEN.
\end{abstract}

Key words: miR-26b; PTEN; ovarian cancer stem cells (CSCs); cell proliferation; apoptosis.

Correspondence: Fang Han, Department of Obstetrics and Gynecology, Foshan Chancheng Central Hospital, 528000 Foshan, Guangdong, China. E-mail: hanfang@gmx.com

Contributions: ZG, XY, contributed equally to this study; FH, YJ, AB study concept; ZG, XY experiment completion; SL, YJ, SH, literature searching; ZG, XY, FH, manuscript drafting and revising; $\mathrm{AB}$, SH, manuscript revising; $\mathrm{AB}, \mathrm{SH}$, data analysis, discussion of results.

Conflict of interest: The authors declare that no conflict of interests exists in this article.

Availability of data and materials: The datasets used and/or analyzed during the current study are available from the corresponding author on reasonable request.

Ethical Approval: All experiments were performed in the Foshan Chancheng Central Hospital and followed the Guidelines. The Ethics Committee of Foshan Chancheng Central Hospital formally approved this study. 


\section{Introduction}

Ovarian cancer (OC) is the most lethal gynecological malignancy with high mortality rate due to the fact that most OC cases are diagnosed in the late stage. ${ }^{1}$ Despite the improvement has been observed in median survival in recent years, most patients still succumb to recurrent, progressive disease due to resistance to chemotherapy. ${ }^{2}$ Thus, there is an urgent need in finding more effective therapeutic options for OC patients. Under this scenario, recent interest has shifted towards ovarian cancer stem cells (CSCs), which are a small subpopulation of tumor cells owning the multilineage differentiation potential and the ability of self-renewal. CSCs have been considered as prime targets for therapeutic intervention. Following these studies, it has been demonstrated that $\mathrm{CD} 44^{+} \mathrm{CD} 117^{+}$cells have the potential to initiate epithelial OC, which was later supported by other studies as well. ${ }^{3}$

Non-coding RNAs are emerging as potential new therapeutic targets in cancer. MicroRNAs (miRNAs) are small non-coding RNAs typically containing 20-24 nucleotides, which play significant roles in many biological processes through sequence-specific regulation of gene expression at the post-transcriptional level. ${ }^{4}$ miRNAs are emerging as important regulators of cancer-related processes, however, the dysregulation of miRNAs in CSCs is still largely unclear. MicroRNA-200c overexpression has been reported to inhibit tumorigenicity and metastasis of $\mathrm{CD} 117^{+} \mathrm{CD} 44^{+}$ovarian CSCs by regulating epithelial-mesenchymal transition. ${ }^{5}$ miRNA23a depletion has also been found to promote apoptosis of ovarian CSC and inhibits cell migration by targeting DLG2. ${ }^{6}$ miR-26 family members have also been verified to suppress cell prolif-eration and tumorigenesis by targeting EZH2 in lung and nasopharyngeal carcinoma. $^{7-9}$ Besides, overexpression of miR-26 has also been revealed to significantly increase the number of apoptotic cells and cause cell cycle arrest in $\mathrm{G}_{0} / \mathrm{G}_{1}$ phase and $\mathrm{G}_{2} / \mathrm{M}$ phase, and miR-26 inhibitor could induce autophagy in human non-small cell lung cancer (NSCLC) A549 cells. ${ }^{10}$ However, the role of miR-26b still remains poorly understood in ovarian CSCs.

Phosphatase and tensin homolog (PTEN) is a well-known tumor suppressor located in 10q23.3, and also the only lipid phosphatase known to counteract the PI3K pathway, and plays critical roles in cancer development. ${ }^{11}$ Loss of PTEN expression represents a common event in various of tumor types. For instance, PTEN loss has been reported to promote intratumoral androgen synthesis and tumor microenvironment remodeling via aberrant activation of RUNX2 in castration-resistant prostate cancer. ${ }^{12}$ Upregulation of PTEN has also been validated to be essential in the anti-miR-19ainhibited cell proliferation and migration in osteosarcoma stem cells. ${ }^{13}$ However, the role of PTEN in the miRNA regulatory network is still incompletely understood in ovarian CSCs. In this study, we unveiled that miR-26b may regulate cell proliferation and apoptosis of $\mathrm{CD} 117^{+} \mathrm{CD} 44^{+}$ovarian CSCs by targeting PTEN. Our data provide new insights into the role of miR-26b and may be an effective therapeutic approach for OC.

\section{Materials and Methods}

\section{Cell line}

The human OC cell lines SKOV3 were commercially available from the China Center for Type Culture Collection (CCTCC, Wuhan, Hubei, China). Cells were maintained and propagated in RPMI-1640 medium containing 10\% fetal bovine serum (FBS) (Gibco, Life Technologies, Grand Island, NY, USA), $100 \mathrm{U} / \mathrm{mL}$ penicillin and $100 \mu \mathrm{g} / \mathrm{mL}$ streptomycin (Sigma-Aldrich, Shanghai,
China) under humidified atmosphere with $5 \% \mathrm{CO}_{2}$ at $37^{\circ} \mathrm{C}$. Once SKOV 3 cells reached $80-90 \%$ confluence, the cells were sub-cultured with the complete media.

\section{Tissue samples}

A total of 15 pairs of OC tissue samples and the adjacent nontumor tissues were obtained from patients with the median age 60 years who received the operation in the Obstetrics and Gynecology Department of the Foshan Chancheng Central Hospital. The tissues were further frozen in liquid nitrogen and stored at $-80^{\circ} \mathrm{C}$ for subsequent analysis. Pathological identification of diagnosis and staging of all the patients was in accordance with the World Health Organization criteria and the Federation International of Gynecology and Obstetrics (FIGO) guidelines on the management of OC. None of the patients had received preoperative chemotherapy or radiation. The process of tissue collection was approved by the ethics committee of Foshan Chancheng Central Hospital.

\section{RNA in situ hybridization (ISH)}

The RNA in situ hybridization was carried out as previously described. ${ }^{14}$ The in situ detection of miR-26b was performed on 6 - $\mu$ m-thick formalin-fixed, paraffin-embedded sections using DIG-labeled miRCURYTM Detection probe (Exiqon, Woburn, MA, USA). Nikon 80i microscope with Nikon NIS-Elements F 2.3 software (Nikon, Shanghai, China) was used to analyze.

\section{$\mathrm{CD}^{117} 7^{+} \mathrm{CD}^{+} 4^{+}$CSCs isolation}

The $\mathrm{CD} 44^{+} \mathrm{CD} 117^{+} \mathrm{CSCs}$ were sorted from the SKOV3 cell line by the immune magnetic-activated cell sorting (MACS, Miltenyi Biotec., Bergisch Gladbach, Germany) based on the previous studies. ${ }^{15,16} \mathrm{CD} 44^{+}$subsets were firstly sorted using mouse anti human CD44 antibody coupled to the magnetic microbeads (Miltenyi Biotec) and followed by the magnetic column selection. Subsequently, mouse anti-human CD117 antibody coupled to magnetic microbeads was used to deplete CD117- subsets (Miltenyi Biotec). The resulting cells are $\mathrm{CD} 44^{+} \mathrm{CD} 117^{+}$, and were placed under stem cell conditions by serum-free DMEM/F12 (Gibco, Life Technologies, Grand Island, NY, USA ), supplemented with 5 $\mu \mathrm{g} / \mathrm{mL}$ insulin (Thermofisher Scientific, Shanghai, China), 20 $\mathrm{ng} / \mathrm{mL}$ human recombinant epidermal growth factor (Thermofisher Scientific), $10 \mathrm{ng} / \mathrm{mL}$ basic fibroblast growth factor (Thermofisher Scientific) and $0.5 \%$ bovine serum albumin (Thermofisher Scientific). ${ }^{17,18}$

\section{Flow cytometry}

The enrichment and recovery of $\mathrm{CD} 44^{+} \mathrm{CD} 117^{+} \mathrm{CSCs}$ were performed on FACS Aria machine (BD Immunocytometry Systems, Franklin Lakes, NJ, USA). Flow cytometry was also used to measure the apoptotic or dead cells by staining with Annexin VFITC and propidium iodide (PI) according to the manufacturer's protocol (Abcam, Cambridge, MA. USA).

\section{qRT-PCR}

Total RNA from the cells was prepared by TRIzol reagent (Invitrogen, Gaithersburg, MD, USA) and quantified by UV-Vis spectroscopy. Then, $2 \mu \mathrm{g}$ of total RNA was subjected to reverse transcription, and $1 \mu \mathrm{L}$ of the product was used for PCR to detect specific gene mRNA level. GAPDH was used as the internal reference gene. For miRNA quantification, the Taqman advanced miRNA cDNA synthesis kit was used to reverse transcription according to the manufacturer's protocols (Applied Biosystems, Carlsbad, CA, USA). Taqman microRNA reverse transcription kit was performed for U6 small nuclear RNA (snRNA) reverse transcription following manufacturer's protocol (Applied Biosystem).

All probes were purchased from Thermofisher Scientific. The 
comparative $2^{-\Delta \Delta C \mathrm{CT}}$ method was used to analyze the relative expression of the target genes and miR-26b. ${ }^{19}$

\section{miR-26b overexpression, inhibition and PTEN upregu- lation}

Negative control oligonucleotides (NC), miR-26b mimics (miR-26b-OE), and PTEN overexpression lentiviral system (PTEN-OE) were commercially available at RiboBio Co. Ltd. (Guangzhou, China). Cells were plated at 30\%-50\% confluence for $24 \mathrm{~h}$. After then, $50 \mathrm{pmol} / \mathrm{mL}$ RNA oligonucleotides were transfected using the Lipofectamine 2000 reagent (Invitrogen) according to the manufacturer's instructions.

\section{3' UTR dual luciferase reporter assay}

We assessed luciferase reporter assay based on the previous study. ${ }^{13}$ Briefly, PTEN 3'-UTR was purchased from Genecopoeia (Rockville, MD, USA; $100 \mathrm{ng} / \mathrm{mL}$ luciferase reporter and 50 $\mathrm{pmol} / \mathrm{mL}$ miR-26b mimics or $\mathrm{NC}$ were co-transfected into the $\mathrm{CD} 117^{+} \mathrm{CD} 44^{+} \mathrm{CSCs}$. After $48 \mathrm{~h}$, luciferase activities were measured using the Dual-Luciferase Reporter Assay System (Promega, Shanghai, China) according to the manufacturer's protocol. The normalization was calculated as the ratio of Firefly luciferase to the Renilla luciferase.

\section{Western blot}

Western blotting was used to detect the protein levels of PTEN. The protein levels of cleaved forms of PARP and caspase-3 (1:500 dilution, Cell Signaling, Shanghai, China) were also detected by Western blot since the common caspase-mediated signaling cascade considered a hallmark of apoptosis.

\section{Cell proliferation assay}

Cells were seeded into 96-well plates (5000 cells/well), and cellular proliferation measurements were carried out by CyQUANT assay kit (Thermofisher Scientific) following the manufacturer's instructions.

\section{Statistics}

All data are represented as the mean $\pm \mathrm{SE}$ and performed for at least three independent experiments. Unpaired Student's $t$-test was carried out for comparison by SPSS software (SPSS Inc. Chicago, IL, USA). The probability values of less than 0.05 were considered significant.
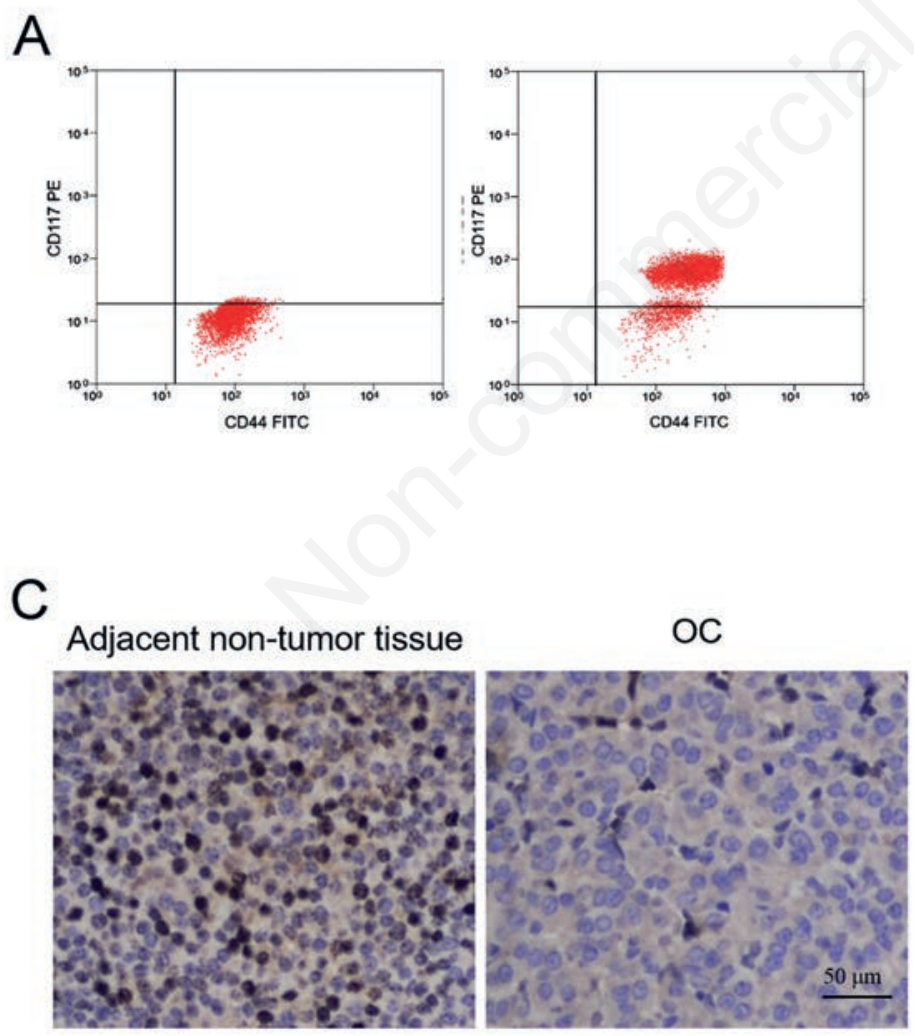

B

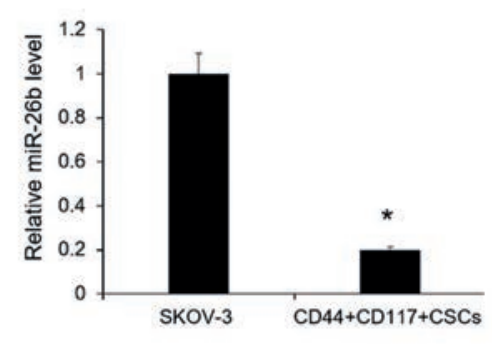

D

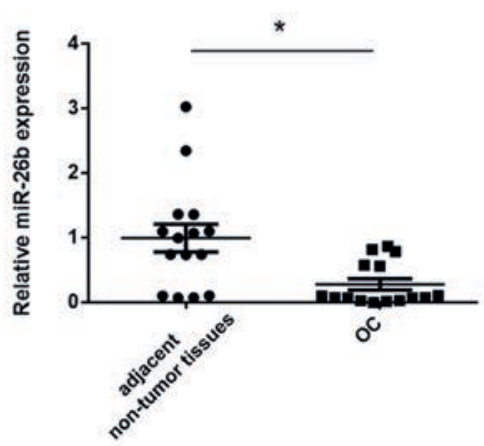

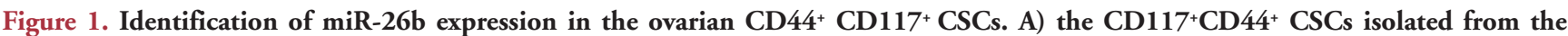
human SKOV3 cell line were validated by FCM; left: SKOV-3 cells; right: CD44+ CD117+CSCs isolated from the SKOV-3 cells. B) miR$26 b$ mRNA level was determined by qRT-PCR. Data are presented as mean $\pm S E$; ${ }^{*} p<0.05$ vs SKOV-3 (n=3). C) representative images of miR-26b expression in OC tissue and adjacent non-tumor tissues were evaluated by ISH staining (200 $\mathrm{x}$ magnification). D) relative miR-26b expression in adjacent non-tumor tissues and OC tissue was measured by $\mathrm{qRT}-\mathrm{PCR} ;{ }^{*} \mathrm{p}<0.05$ vs adjacent non-tumor tissues. 


\section{Results}

\section{miR-26b is under expressed in $\mathrm{CD}^{4} 4^{+} \mathrm{CD} 117^{+} \mathrm{CSCs}$}

The $\mathrm{CD} 44^{+} \mathrm{CD} 117^{+} \mathrm{CSC}$ s were isolated from the SKOV-3 cell line using MACS, and flow cytometry assay was performed by analysis the percentage of the $\mathrm{CD} 44^{+} \mathrm{CD} 117^{+}$cells in SKOV3 after enriched in the stem cell culture medium for 2 days. We found that flow cytometry percent positive cells for CD44 and CD117 was around $82.5 \%$ after enrichment compared with $4.3 \%$ in the SKOV3 cells (Figure 1A).

To investigate the role of miR-26b in $\mathrm{CD} 44^{+} \mathrm{CD} 117^{+} \mathrm{CSCs}$, its expression level in $\mathrm{CD} 44^{+} \mathrm{CD} 117^{+} \mathrm{CSC}$ s was determined by qRTPCR, and the results showed that the expression of miR-26b was strongly lower in the $\mathrm{CD} 44^{+} \mathrm{CD} 117^{+} \mathrm{CSC}$ s than that in the SKOV3 cells $(p<0.05)$ (Figure 1B). Furthermore, ISH staining (Figure 1C) was used to establish the pathologic and clinical significance of miR-26b expression in OC, which was carried out by the pathology department. We also detected the expression levels of miR-26b in 15 OC tissue samples and related non-tumor tissues using qRTPCR. As shown in Figure 1D, the expression levels of miR-26b in $\mathrm{OC}$ cancer tissues were strongly lower than the adjacent non-tumor tissues.

\section{miR-26b overexpression inhibits the cell proliferation and promotes cell apoptosis}

To validate the biological function of miR-26b, we overex- pressed miR-26b in $\mathrm{CD}_{4} 4^{+} \mathrm{CD} 117^{+} \mathrm{CSCs}$ by transfection with miR-26b. As shown in Figure 2A, miR-26b was significantly increased in $\mathrm{CD} 44^{+} \mathrm{CD} 117^{+} \mathrm{CSCs}$ with miR-26b overexpression. We next assessed cell proliferation using the Cyquant assay. We observed that the cell growth in the miR-26b overexpression group was significantly suppressed from day 3 in contrast to the NC and SKOV-3 cell groups (Figure 2B). Moreover, Annexin V and PI staining was applied to determine the apoptotic cells and the results showed that the apoptotic process in the miR-26b overexpression group $(30.5 \%)$ was strongly promoted compared with the $\mathrm{NC}$ and SKOV-3 cell groups (45.7\%) (Figure 2C). Furthermore, cleaved PARP and caspase-3 (i.e., apoptosis markers) were increased in cells overexpressing miR-26b (Figure 2D).

\section{PTEN is repressed by miR-26b}

In order to decipher the underlying molecular mechanism of how miR-26b regulates cell proliferation and apoptosis, we subsequently identified the target genes of miR-26b. PTEN has been reported as the direct target gene of miR-26b in other diseases. ${ }^{20}$ Furthermore, as we observed that miR-26b was downregulated in $\mathrm{CD} 44^{+} \mathrm{CD} 117^{+} \mathrm{CSCs}$ (Figure 1B), the expression of PTEN was found to be significantly increased in $\mathrm{CD} 44^{+} \mathrm{CD} 117^{+} \mathrm{CSC}$ compared with that in the SKOV-3 cells (Figure 3A). Moreover, the expression level of PTEN was also found to be remarkably increased in OC tissues compared with the adjacent non-tumor tissues (Figure 3B).

The 3' UTR luciferase assay was performed to validate the
A

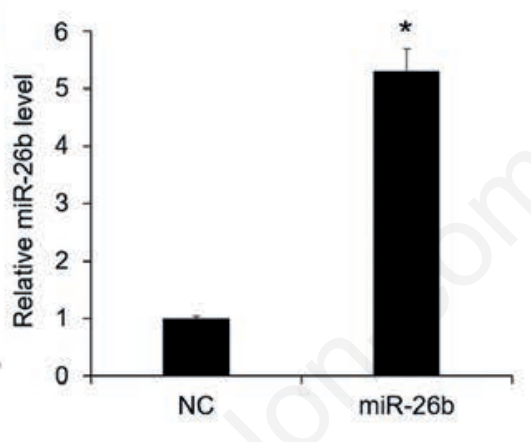

B

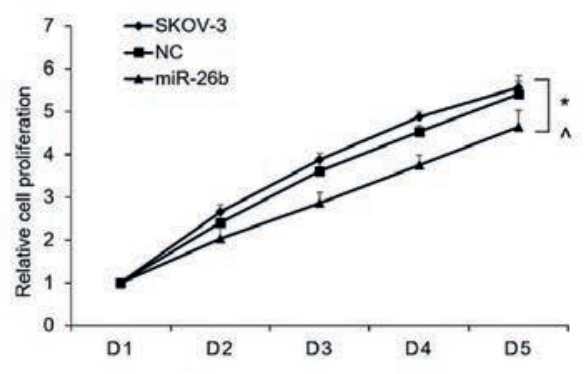

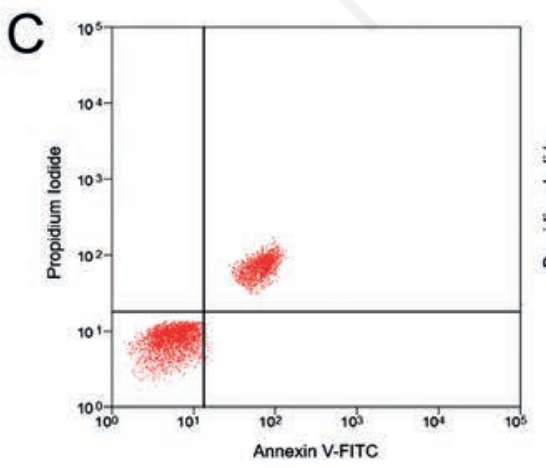
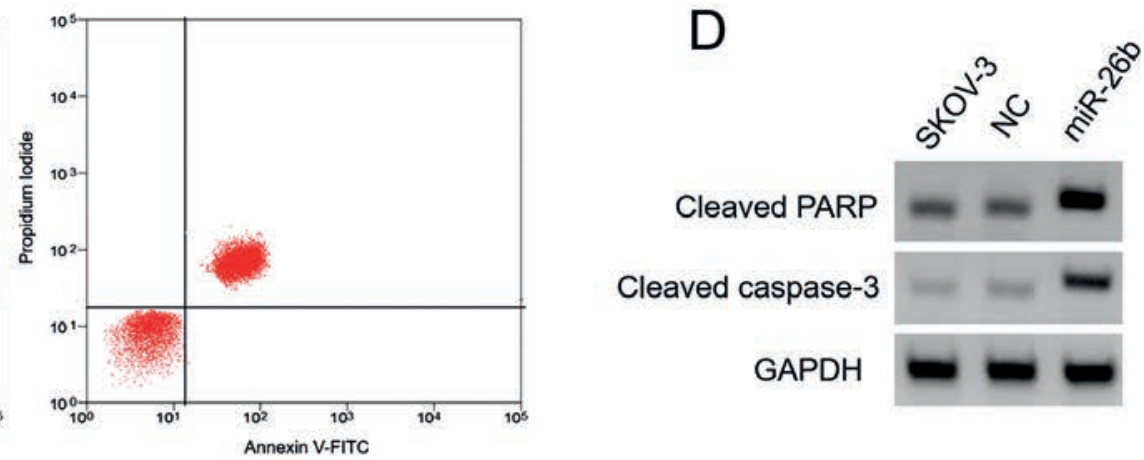

Figure 2. miR-26b inhibits cell proliferation and promotes cell apoptosis in CD117 ${ }^{+} \mathrm{CD} 44^{+}$CSCs. A) miR-26b mRNA level in

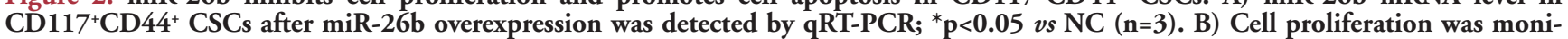
tored by Cyquant assay after miR-26b overexpression; ${ }^{*} \mathrm{p}<0.05$ vs NC $(\mathrm{n}=3),{ }^{\wedge} \mathrm{p}<0.05$ vs SKOV-3 (n=3). C) Cell apoptosis was analyzed by flow cytometry after miR-26b transfection; left: SKOV-3 cells overexpressed with NC; right: SKOV-3 cells overexpressed with miR26b. D) Western blot showing increased apoptotic proteins (cleaved PARP and caspase-3) in CD117 ${ }^{+}$CD $44^{+}$CSCs overexpressing miR26b. Data are presented as mean \pm SE. 
negative relationship between miR-26b and PTEN in $\mathrm{CD} 44^{+} \mathrm{CD} 117^{+} \mathrm{CSCs}$. We found that miR-26b overexpression but not NC significantly decreased the luciferase reporter activity of PTEN 3' UTR (Figure 3C). In addition, we also found that the protein level of PTEN was reduced after cells overexpressed with miR-26b compared with the NC and non-treatment cells (Figure 3D).

\section{miR-26b regulates $\mathrm{CD} 44^{+} \mathrm{CD} 117^{+} \mathrm{CSCs}$ through PTEN}

To explore the role of PTEN in the miR-26b regulated cell proliferation and apoptosis, we overexpressed PTEN in the cells transfected with miR-26b. The overexpression of PTEN was confirmed in both mRNA and protein levels (Figure 4 A,B). We observed that PTEN overexpression reversed the effects of miR-26b on cell proliferation and apoptosis. PTEN overexpression remarkably accelerated cell proliferation, and inhibited cell apoptosis (Figure 4 C,D). The apoptotic cells in the PTEN overexpression group $(43.9 \%)$ were strongly inhibited compared with the control group (25.7\%) (Figure 4D). In addition, cleaved PARP and caspase-3 were also downregulated in cells overexpressing PTEN after miR$26 \mathrm{~b}$ knockdown (Figure 4E). This data suggests that miR-26b regulates cell proliferation and apoptosis in $\mathrm{CD} 44^{+} \mathrm{CD} 117^{+} \mathrm{CSCs}$ through PTEN.

\section{Discussion}

Accumulating researches have demonstrated that CSCs are a small subpopulation of cancer cells characterized with self-renewal, multilineage differentiation potential, and tumorigenicity. ${ }^{21}$ To understand the exact biological features of OC, numerous studies are being focused on CSCs due to their ability of tumor initiation and self-renewal. Thus, CSCs have been suggested as a promising target for cancer therapy. In spite of that, whether the CSCs are neoplastic counterpart of a normal stem cell or whether complete malignant behaviour can occur in a more differentiated cell has still to be demonstrated. ${ }^{22}$ Recently, studies of CSCs reported that miRNAs play important roles in the progression of CSCs. ${ }^{6,23}$ However, it is still not much known about whether and how miR$26 \mathrm{~b}$ and its regulatory network are involved in ovarian CSCs.

miR-26b has been reported to play critical roles in multiple tumors. For instance, miR-26b-5p has been reported to help in EpCAM $^{+}$CSCs maintenance via HSC71/HSPA8 and augments malignant features in $\mathrm{HCC} .{ }^{24} \mathrm{miR}-26 \mathrm{~b}-5 \mathrm{p}$ has also been validated to inhibit the proliferation of bladder can cells by regulating PDCD $10 .{ }^{25}$ In addition, miR-26b promotes colorectal cancer metastasis by downregulating PTEN and wingless-type MMTV integration site family member $5 \mathrm{~A} .{ }^{26}$ However, the role of miR$26 \mathrm{~b}$ is still remains poorly understood in ovarian CSCs.

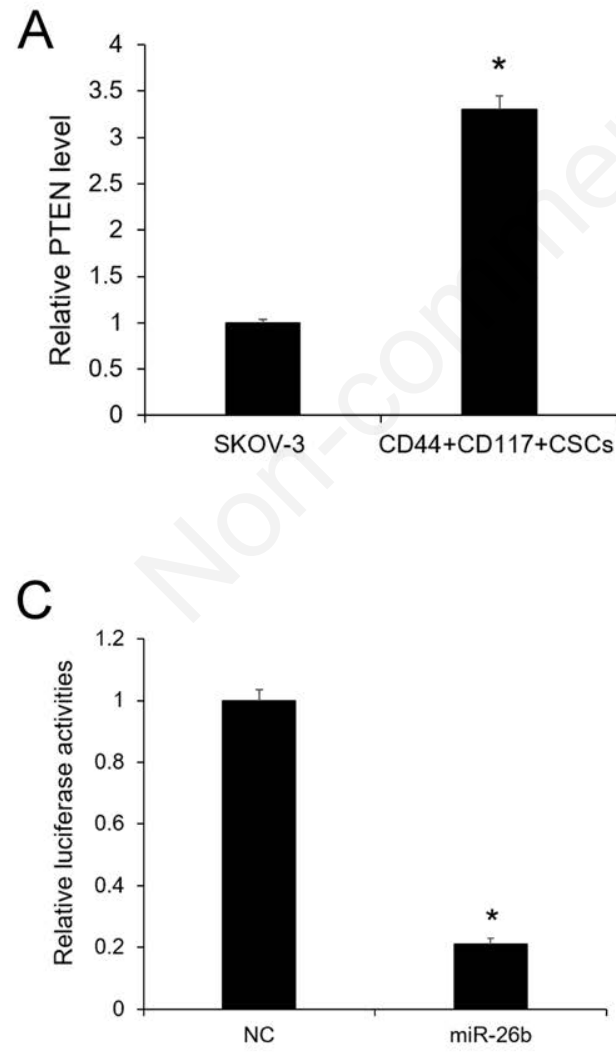

B

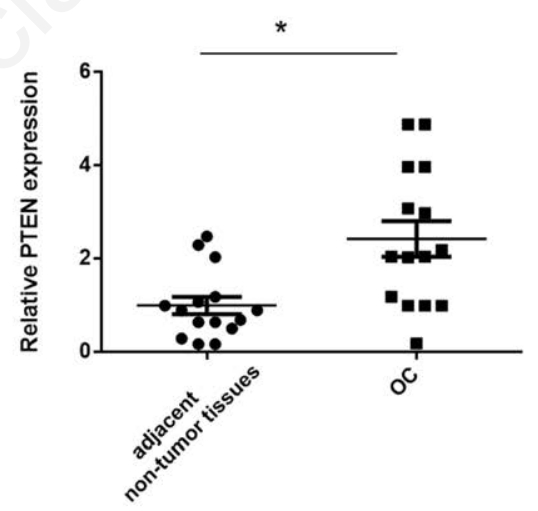

D

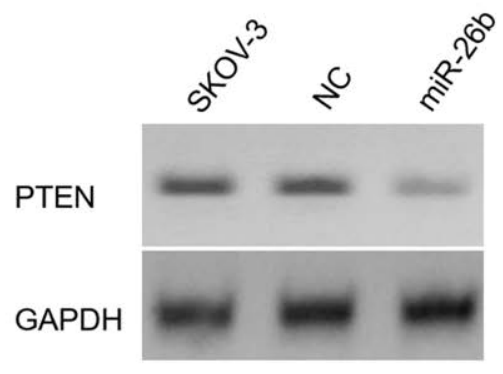

Figure 3. PTEN is the direct target of miR-19a in CD117 ${ }^{+}$CD $44^{+}$CSCs. A) PTEN mRNA level was detected by $\mathrm{qRT}-\mathrm{PCR}$; ${ }^{\mathrm{p}}<0.05$ vs SKOV-3 (n=3). B) relative PTEN expression in adjacent non-tumor tissues and OC tissue was measured by $\mathrm{qRT}-\mathrm{PCR}$; ${ }^{\mathrm{p}<0.05} \boldsymbol{v s}$ adjacent non-tumor tissues. C) The luciferase activity in CD117 ${ }^{+} \mathrm{CD} 44^{+} \mathrm{CSC}$ s was analyzed by Dual-Luciferase Reporter Assay System following the manufacturer's protocol; ${ }^{*} \mathrm{p}<0.05 v s \mathrm{NC}(\mathrm{n}=3)$. D) after transfection with miR-26b mimics for $48 \mathrm{~h}$, the expression of PTEN at protein level in $\mathrm{CD} 117^{+} \mathrm{CD} 44^{+} \mathrm{CSC}$ s was measured by Western blot. Data are presented as mean \pm SE. 

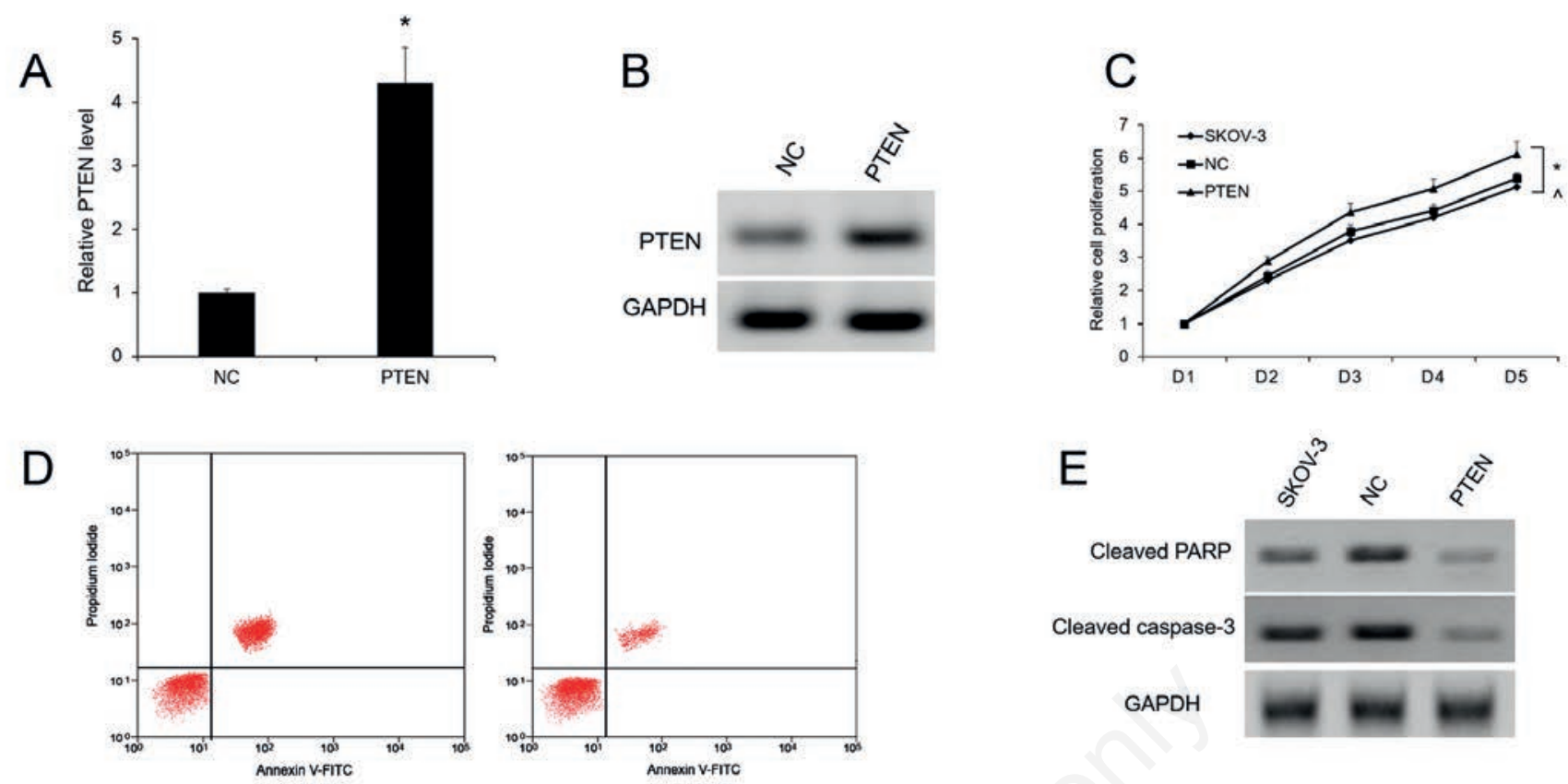

Figure 4. PTEN overexpression accelerates the cell proliferation and suppresses cell apoptosis in CD117 $7^{+}$CD44 ${ }^{+}$CSCs. A,B) PTEN mRNA level and protein level were detected by $q R T-P C R$ in miR-26b overexpressed CD117 ${ }^{+}$CD $44^{+}$CSCs with PTEN upregulation; ${ }^{*} \mathrm{p}<0.05$ vs NC (n=3). C) Cell proliferation was measured by Cyquant assay after in miR-26b overexpressed $\mathrm{CD} 117^{+} \mathrm{CD} 44^{+} \mathrm{CSC}$ with PTEN upregulation; * $\mathrm{p}<0.05$ vs $\mathrm{NC}(\mathrm{n}=3),{ }^{\wedge} \mathrm{p}<0.05$ vs SKOV-3 $(\mathrm{n}=3)$. D) Cell apoptosis was analyzed by flow cytometry after PTEN transfection; left: SKOV-3 cells overexpressed with NC; right: SKOV-3 cells overexpressed with PTEN. E) Apoptotic proteins (cleaved PARP and caspase-3) were determined by Western blot in in miR-26b overexpressed CD117 ${ }^{+}$CD44 ${ }^{+}$CSCs with PTEN upregulation. Data are presented as mean \pm SE.

PTEN is a well-known tumor suppressor, and usually underexpressed in different types of tumors. The dysfunction of PTEN may lead to critical outcomes in in tumor initiation and development. PTEN has been validated to inhibit the formation of CSCs by inhibiting the PI3K/Akt pathway in prostate cancer. ${ }^{27}$ MiRNAs/PTEN axis has also been proved to regulate the cell progress in multiple tumors. IL- $1 \beta / \mathrm{NF}-\mathrm{kb}$ signaling has been revealed to promote colorectal cancer cell growth through miR$181 \mathrm{a} / \mathrm{PTEN}$ axis. ${ }^{28}$ Moreover, miR-19a has been confirmed to regulate cell growth and apoptosis of osteosarcoma stem cells by targeting PTEN. ${ }^{13}$ However, the role of miR-26b/PTEN axis is still incompletely understood in ovarian CSCs.

In this study, we proved that miR-26b was downregulated in ovarian $\mathrm{CD} 44^{+} \mathrm{CD} 117^{+} \mathrm{CSCs}$. In addition, we also found that miR26b overexpression inhibited cell proliferation and promoted cell apoptosis. Moreover, PTEN was validated as the direct target gene of miR-26b and PTEN overexpression reversed the effect of miR$26 \mathrm{~b}$ on cell proliferation and apoptosis. These results allow suggesting that miR-26b regulates the proliferation and apoptosis of $\mathrm{CD} 44^{+} \mathrm{CD} 117^{+} \mathrm{CSC}$ through suppressing PTEN, which also provide potential biological mechanism on drug resistance. However, to confirm this hypothesis, cellular experiments should be conducted in PTEN knock-down/out cells. Further studies should also be performed to measure the percentage of CSCs in OC, and investigate the effect of miR-26b on stemness of OC cells and the downstream effectors of PTEN. Moreover, the impeded effect alternation after co-transfected with miR-26b and PTEN in CSCs and in vivo testing should also be investigated extensively. Once miR26b/PTEN axis role had been confirmed in vivo, miR-26b/PTEN would become a novel therapeutic target for OC patients.

\section{References}

1. Grzelak MM, Wrobel PM, Lankosz M, Stegowski Z, Chmura L, Adamek D, et al. Diagnosis of ovarian tumour tissues by SR-FTIR spectroscopy: A pilot study. Spectrochim Acta A Mol Biomol Spectrosc 2018;203:48-55.

2. Vargas-Hernandez VM, Moreno-Eutimio MA, AcostaAltamirano G, Vargas-Aguilar VM. Management of recurrent epithelial ovarian cancer. Gland Surg 2014;3:198-202.

3. Zhang S, Balch C, Chan MW, Lai HC, Matei D, Schilder JM, et al. Identification and characterization of ovarian cancer-initiating cells from primary human tumors. Cancer Res 2008;68:4311-20.

4. Zhao B, Yu Q, Li H, Guo X, He X. Characterization of microRNA expression profiles in patients with intervertebral disc degeneration. Int J Mol Med 2014;33:43-50.

5. Chen D, Zhang Y, Wang J, Chen J, Yang C, Cai K, et al. MicroRNA-200c overexpression inhibits tumorigenicity and metastasis of CD117+CD44+ ovarian cancer stem cells by regulating epithelial-mesenchymal transition. J Ovarian Res 2013;6:50.

6. Zhuang RJ, Bai XX, Liu W. MicroRNA-23a depletion promotes apoptosis of ovarian cancer stem cell and inhibits cell migration by targeting DLG2. Cancer Biol Ther 2019;20:897911.

7. Dang X, Ma A, Yang L, Hu H, Zhu B, Shang D, et al. MicroRNA-26a regulates tumorigenic properties of EZH2 in human lung carcinoma cells. Cancer Genet 2012;205:113-23.

8. Lu J, He ML, Wang L, Chen Y, Liu X, Dong Q, et al. miR-26a inhibits cell growth and tumorigenesis of nasopharyngeal car- 
cinoma through repression of EZH2. Cancer Res 2011;71:22533.

9. Sun J, Tian X, Lu SQ, Hu HB. MicroRNA-4465 suppresses tumor proliferation and metastasis in non-small cell lung cancer by directly targeting the oncogene EZH2. Biomed Pharmacother 2017;96:1358-62.

10. He Y, Liu H, Jiang L, Rui B, Mei J, Xiao H. miR-26 Induces apoptosis and inhibits autophagy in non-small cell lung cancer cells by suppressing TGF-beta1-JNK signaling pathway. Front Pharmacol 2018;9:1509.

11. Papa A, Pandolfi PP. The PTEN(-)PI3K axis in cancer. Biomolecules 2019;9:153.

12. Yang Y, Bai Y, He Y, Zhao Y, Chen J, Ma L, et al. PTEN loss promotes intratumoral androgen synthesis and tumor microenvironment remodeling via aberrant activation of RUNX2 in castration-resistant prostate cancer. Clin Cancer Res 2018;24:834-46.

13. Zhao D, Chen Y, Chen S, Zheng C, Hu J, Luo S. miR-19a regulates the cell growth and apoptosis of osteosarcoma stem cells by targeting PTEN. Tumour Biol 2017;39:10104283177053 41.

14. Dong YX, Pang ZG, Zhang JC, Hu JQ, Wang LY. Long noncoding RNA GClnc1 promotes progression of colorectal cancer by inhibiting p53 signaling pathway. Eur Rev Med Pharmacol Sci 2019;23:5705-13.

15. Chen J, Wang J, Chen D, Yang J, Yang C, Zhang Y, et al. Evaluation of characteristics of CD $44+\mathrm{CD} 117+$ ovarian cancer stem cells in three dimensional basement membrane extract scaffold versus two dimensional monocultures. BMC Cell Biol 2013;14:7.

16. Dou J, Pan M, Wen P, Li Y, Tang Q, Chu L, et al. Isolation and identification of cancer stem-like cells from murine melanoma cell lines. Cell Mol Immunol 2007;4:467-72.

17. Arnhold S, Gluer S, Hartmann K, Raabe O, Addicks K, Wenisch S, et al. Amniotic-fluid stem cells: Growth dynamics and differentiation potential after a CD-117-based selection procedure. Stem Cells Int 2011;2011:715341.

18. Luo L, Zeng J, Liang B, Zhao Z, Sun L, Cao D, et al. Ovarian cancer cells with the CD117 phenotype are highly tumorigenic and are related to chemotherapy outcome. Exp Mol Pathol 2011;91:596-602.

19. Yin Q, Fischer L, Noethling C, Schaefer WR. In vitro-assessment of putative antiprogestin activities of phytochemicals and synthetic UV absorbers in human endometrial Ishikawa cells. Gynecol Endocrinol 2015;31:578-81.

20. Zhang L, Huang C, Guo Y, Gou X, Hinsdale M, Lloyd P, et al. MicroRNA-26b modulates the NF-kappaB pathway in alveolar macrophages by regulating PTEN. J Immunol 2015; 195:5404-14.

21. Kreso A, Dick JE. Evolution of the cancer stem cell model. Cell Stem Cell 2014;14:275-91.

22. Vezzoni L, Parmiani G. Limitations of the cancer stem cell theory. Cytotechnology 2008;58:3-9.

23. Xia Q, Han T, Yang P, Wang R, Li H, Zhang J, et al. MicroRNA-28-5p regulates liver cancer stem cell expansion via IGF-1 pathway. Stem Cells Int 2019;2019:8734362.

24. Khosla R, Hemati H, Rastogi A, Ramakrishna G, Sarin SK, Trehanpati N. miR-26b-5p helps in EpCAM+cancer stem cells maintenance via HSC71/HSPA8 and augments malignant features in HCC. Liver Int 2019;39:1692-703.

25. Wu K, Mu XY, Jiang JT, Tan MY, Wang RJ, Zhou WJ, et al. miRNA26a5p and miR26b5p inhibit the proliferation of bladder cancer cells by regulating PDCD10. Oncol Rep 2018;40:3523-32.

26. Fan D, Lin X, Zhang F, Zhong W, Hu J, Chen Y, et al. MicroRNA 26b promotes colorectal cancer metastasis by downregulating phosphatase and tensin homolog and wingless-type MMTV integration site family member 5A. Cancer Sci 2018;109:354-62.

27. Kim RJ, Bae E, Hong YK, Hong JY, Kim NK, Ahn HJ, et al. PTEN loss-mediated Akt activation increases the properties of cancer stem-like cell populations in prostate cancer. Oncology 2014;87:270-9.

28. Hai Ping P, Feng Bo T, Li L, Nan Hui Y, Hong Z. IL-1beta/NF$\mathrm{kb}$ signaling promotes colorectal cancer cell growth through miR-181a/PTEN axis. Arch Biochem Biophys 2016;604:20-6.

Received for publication: 4 October 2020. Accepted for publication: 4 January 2021.

This work is licensed under a Creative Commons Attribution-NonCommercial 4.0 International License (CC BY-NC 4.0).

CCopyright: the Author(s), 2021

Licensee PAGEPress, Italy

European Journal of Histochemistry 2021; 65:3186

doi:10.4081/ejh.2021.3186

[European Journal of Histochemistry 2021; 65:3186] 